\title{
Hemoglobin Expression and Function in Human Corneal Epithelium
}

\author{
Ryan McBride ${ }^{1}$, Erin Perez ${ }^{1}$, Ernest Talarico ${ }^{1}$, Brian Kennedy ${ }^{1}$ \\ Indiana University School of Medicine Northwest - Gary, IN
}

The corneal epithelium forms the outer layer of the cornea. It provides mechanical protection, prevents fluid loss and forms a barrier to invasive pathogens. Though hemoglobin expression has been extensively studied in erythroid cells, recent work in multiple cell systems has documented hemoglobin expression in cells of non-erythroid origin. However, the function of hemoglobin in non-erythroid cells has yet to be established. The hypothesis that hemoglobin is expressed in corneal epithelium and that it functions to protect against oxidative stress will be examined in the present work. Hemoglobin expression and function was examined by immunocytochemistry and western blot analysis. Native human corneal explants and an immortalized human corneal epithelial cell line were examined. Expression of hemoglobin beta and delta chains was demonstrated at the protein level in both native and cell culture preparations. Hexamethylene bisacetamide is a known inducer of hemoglobin beta chain expression in murine erythroleukemia cells. HMBA did function to increase beta chain expression in cultured corneal epithelium as well. Beta chain immunolocalization was primarily cytoplasmic, while the delta chain localized to both cytoplasmic and membrane domains. Oxidative stress, from hydrogen peroxide exposure, was shown to upregulate delta chain expression. In conclusion, hemoglobin chains are expressed in corneal epithelium and could function to protect against oxidative stress. This is relevant given that exposure to light and high oxygen tensions render the cornea particularly susceptible to oxidative damage. 